\title{
Active gingival inflammation is linked to Hypertension
}

Short title: Gingival bleeding and hypertension

Authors: Davide PIETROPAOLI ${ }^{\text {a }}$, Annalisa MONACO a, Francesco D'AIUTO ${ }^{\text {b }}$, Eva MUÑOZ AGUILERA ${ }^{\text {b }}$, Eleonora ORTU a, Mario GIANNONI a, Marta CZESNIKIEWICZ-GUZIK ${ }^{\mathrm{c}, \mathrm{d}}$, Tomasz J. GUZIK e,f $^{\text {,f }}$ Claudio FERRI ${ }^{\text {, }}$, Rita DEL PINTO ${ }^{g}$ *

* These Authors contributed equally to this paper.

\section{Affiliations:}

a Department of Life, Health and Environmental Sciences, San Salvatore Hospital, University of L'Aquila, - Unit of Dentistry - Center for Oral Diseases and Translational Research - Oral DISeases and SYstemic interactions study group (ODISSY group), L'Aquila, Italy.

b Periodontology Unit, UCL Eastman Dental Institute and Hospital, University College London, London, UK.

c Department of Periodontology and Oral Sciences Research Group, University of Glasgow Dental School, Glasgow, UK.

${ }^{d}$ Department of Dental Prophylaxis and Experimental Dentistry, Jagiellonian University, Collegium Medicum, Krakow, PL

e Institute of Cardiovascular and Medical Sciences, University of Glasgow, Glasgow, UK.

${ }^{\mathfrak{f}}$ Department of Internal and Agricultural Medicine, Jagiellonian University, Collegium Medicum, Krakow, PL

${ }^{g}$ Department of Life, Health and Environmental Sciences, San Salvatore Hospital, University of L'Aquila, - Unit of Internal Medicine and Nephrology, Center for Hypertension and Cardiovascular Prevention - Oral DISeases and SYstemic interactions study group (ODISSY group), L'Aquila, Italy. 


\section{Corresponding author:}

Dr. Davide Pietropaoli

University of L'Aquila

Dept. of Life Health and Environmental Sciences

San Salvatore Hospital - Bldg Delta 6, Floor -1, Room \#21

Via Petrini 67100 - L’Aquila

Email: davide.pietropaoli@univaq.it

Phone: +390862 434974

Fax: +390862434974

Word count: 4260 please list full word count (including references, but not tables and legends)

Number of figures: 1

Number of tables: 2

Number of supplementary digital content files:

- 10 Supplementary Tables

- 3 Supplementary Figures 


\section{Abstract}

Background. Cardiovascular diseases (CVD) including hypertension, are characterized by underlying systemic inflammation. Periodontitis which can impact the systemic inflammatory burden has recently been linked to high blood pressure (BP). However, the relationship of gingival bleeding, as an easily accessible marker of periodontal pathology, with hypertension, remains unclear.

Methods. Survey-based propensity score matching (PSM) incorporating major confounders shared between hypertension and periodontal diseases was applied to cross-sectional NHANES III data from 5.396 adults $\geq 30$ years who underwent BP measurement and periodontal examination, identifying two matched groups with and without gingival bleeding. The association of bleeding gums with systolic BP $(\mathrm{mmHg})$ and high/uncontrolled BP was then assessed with generalized additive models incorporating inflammatory markers. Stratification by periodontal status (healthy; gingivitis; stable periodontitis; unstable periodontitis) was performed. Variables importance was estimated using machine learning.

Results. Gingival bleeding (gingivitis; unstable periodontitis) was independently associated with $+2.6 \mathrm{mmHg}(\mathrm{p}<0.001)$ systolic BP compared with no bleeding (healthy periodontium; stable periodontitis), and with greater odds (OR=1.42;95\%C.I.=1.19-1.68; $\mathrm{p}<0.001)$ of high/uncontrolled BP. Participants with unstable periodontitis had higher systolic BP than those with stable periodontitis $(+2.1 \mathrm{mmHg} ; \mathrm{p}<0.001)$ or gingivitis $(+5.3 \mathrm{mmHg} ; \mathrm{p}<0.001)$. Unstable periodontitis and gingivitis were consistently associated with increased risk of high/uncontrolled BP $(O R=1.65$, 95\%C.I.=2.14-1.82; OR=1.49, 95\%C.I.=1.22-1.82, respectively). Inflammatory markers allowed a maximum of $12 \%$ gain in the models' predictive power.

Conclusions. Gingival bleeding contributes to shaping the relationship between periodontal diseases and $\mathrm{BP}$, but the burden represented by periodontitis is also crucial. Periodontal evaluation might be of importance in difficult to control hypertension.

Keywords: Hypertension; Inflammation; Periodontal Disease; Gingivitis; Periodontal Index 


\section{Introduction}

Cardiovascular (CV) prevention is of paramount importance for the reduction of morbidity and mortality worldwide [1]. Population interventions as well as drug therapies addressing metabolic imbalance play a pivotal role [1]. High blood pressure (BP), in particular, represents a global epidemic that was estimated to cause 10 million premature CV deaths and over 200 million disability-adjusted life years in 2015 (+40\% compared to 1990) [2]. Importantly, a considerable proportion of individuals treated for high BP still fails to reach therapeutic goals in spite of antihypertensive treatment [3-5].

Besides traditional CV risk factors [4], systemic inflammation has emerged as a consistent contributor to CV disease (CVD) burden and more recently to hypertension [3-5]. Of notice, periodontal diseases, i.e. those affecting the soft and hard tissues surrounding the teeth, can have a significant impact on systemic inflammation [6,7]. They are estimated to affect over $50 \%$ of the population worldwide and to be the $6^{\text {th }}$ most prevalent disease of humankind $[8,9]$. The clinical spectrum of periodontal diseases includes gingivitis and periodontitis, and is characterized by a relapsing-remitting behaviour, whose clinical hallmark is represented by gingival bleeding [10]. The extent of the inflamed area during active disease is estimated to approximately equal the size of a palm of the hand in severe cases [11]. Gingival bleeding has been linked to increased systemic inflammatory burden compared to healthy gums and stable periodontitis [12-14]. Moreover, bleeding on probing $(\mathrm{BoP})$ is routinely assessed and considered a valuable clinical parameter of periodontal inflammation, which, in turn, may give rise to systemic inflammatory conditions, including CV disorders [15,16].

Although the association between periodontal disorders and BP has recently been documented [17-19], the role of gingival bleeding in this context has been sparsely investigated. This is an important aspect as gingival bleeding can be considered a widely available marker of periodontal health status $[10,20]$. Specifically, whether the risk of high/uncontrolled BP may vary throughout the natural history of periodontal diseases, i.e. if it is modified by gingival bleeding, is unclear. 
Herein, we tested whether presence of bleeding gums is associated with high/uncontrolled BP across the clinical spectrum of periodontal disorders after controlling for major shared confounders, and how inflammatory markers impact on this association. 


\section{Methods}

\section{$\underline{\text { Data Source }}$}

NHANES III data are accessible through the Centers for Disease Control and Prevention (CDC) National Center for Health Statistics (NCHS) website at https://www.cdc.gov/nchs/nhanes/index.htm. This study was deemed exempt from review by local Institutional Review Boards.

\section{Study population}

The study population was derived from NHANES III dataset following the procedure described in the statistical analysis paragraph. NHANES III was a nationwide probability sample of 39,695 civilian non-institutionalized US persons aged 2 months and older, conducted from 1988 to 1994 [21]. The population selected for this analysis includes NHANES III participants aged 30 years and older, with complete periodontal evaluation in terms of BoP, periodontal probing depth (PPD) and clinical attachment loss (CAL) and with available and valid BP measurements. The age threshold reflects age requirements for the estimation of the prevalence of periodontitis according to the current definition of the disease used in epidemiological studies [22,23].

Oral health data collection protocols were approved by the CDC, NCHS Research Ethics Review Board, Atlanta (USA), and all survey participants provided written informed consent. All the examinations were conducted in a mobile examination center.

\section{$\underline{\text { Periodontal examination }}$}

Participants who did not meet any of the exclusion criteria listed in the NHANES III protocol (a history of congenital heart disease, heart valve disease, pacemaker or other artificial cardiac devices, bacterial endocarditis, rheumatic fever, kidney disease requiring dialysis, hemophilia, or bone/joint prosthesis https://wwwn.cdc.gov/nchs/data/nhanes3/manuals/dental.pdf) [24] were eligible for periodontal examination, consisting of two-sites (mid-facial and mesio-facial) assessment of PPD, CAL, and BoP for each tooth in two quadrants, one maxillary and one mandibular, randomly chosen by a computer function on the assumption that the relative 
conditions would be representative of the oral health status. Third molars were excluded from analysis because of their frequent extraction in young adulthood, so a maximum of 14 teeth and 28 sites per individual could be examined. Pregnant women were excluded due to potential risk for both gingivitis and pre-eclampsia.

\section{Definition of periodontal health status and disease activity}

Presence of periodontitis was defined according to case definition for population-based surveillance that was employed in NHANES, i.e. as $\geq 2$ interproximal sites with CAL $\geq 3 \mathrm{~mm}$ and $\geq 2$ interproximal sites with PPD $\geq 4 \mathrm{~mm}$ (not on the same tooth), or one site with PPD $\geq 5 \mathrm{~mm}$ [23], while disease stability was defined as documented gingival bleeding in terms of $\mathrm{BoP}<10 \%$ according to recent guidelines [10,20]. Patients were stratified according to the presence of bleeding gums and to their periodontal status. Specifically, the dichotomous status of non-bleeding or bleeding gums was determined according to BoP (non-bleeding gums: $<10 \%$ of sites with BoP; bleeding gums: $\geq 10 \%$ of sites with BoP), irrespective of the presence of periodontitis $[10,20]$. Periodontal status, indeed, was defined as: 1) "healthy", in the absence of both periodontitis and bleeding gums (BoP<10\%); 2) "gingivitis", in the presence of gingival bleeding (BoP $\geq 10 \%)$, but without signs of periodontitis; 3 ) "stable periodontitis", in the presence of clinical signs of periodontitis, but without bleeding gums (BoP<10\%); and 4) "unstable periodontitis", with concurrent presence of signs of periodontitis and gingival bleeding $(\mathrm{BoP} \geq 10 \%)[10,20]$. BoP was also used as a continuous variable (\%). In addition, periodontal inflamed surface area (PISA, $\mathrm{mm}^{2}$ ) was calculated for each patient with a custom-made function $[11,25]$.

\section{Blood pressure measurement and classification}

Arterial BP was measured by trained and calibrated personnel using a mercury sphygmomanometer according to standardized BP measurement protocols. For this analysis, we used the average (mean \pm standard deviation, SD) of three consecutive BP readings taken for each patient on the same arm. 
According to a previous approach $[19,25]$, BP was then modeled in two different ways: 1) continuous variable, as systolic BP ( $\mathrm{mmHg}$ ), given its stronger association with periodontal disorders than diastolic BP [26]; 2) dichotomous variable, as BP status/control (<130/80 mmHg: normal/controlled BP; $\geq 130 / 80 \mathrm{mmHg}$ : high/uncontrolled BP). In accordance with the American College of Cardiology/American Heart Association (ACC/AHA) 2017 Guidelines for the Prevention, Detection, Evaluation, and Management of High Blood Pressure in Adults, this threshold identifies the diagnosis of hypertension in untreated patients, and the achievement of treatment goal in those receiving BP medications [27]. An additional definition of high/uncontrolled BP was also tested, based on the 2018 European Society of Cardiology/European Society of Hypertension (ESC/ESH) guidelines for the management of hypertension $(\geq 140 / 90 \mathrm{mmHg})[5]$.

\section{$\underline{\text { Classification of covariates }}$}

Additional data was used for descriptive and inferential statistics, as appropriate. In particular, participants were stratified by categories of age (30-44, 45-64, and $\geq 65$ years), gender, race (nonHispanic Whites; non-Hispanic Blacks; Mexican-Americans; other), ethnicity (Hispanics; nonHispanics), glycemic status (normoglycemia, pre-diabetes, or diabetes mellitus based on glycohemoglobin $\mathrm{A} 1 \mathrm{c}[\mathrm{HbA} 1 \mathrm{c}]<5.7 \%, 5.7 \%-6.4 \%$, or $\geq 6.5 \%$, respectively), body mass index (BMI) (underweight, normal weight, overweight, or obese based on $\mathrm{BMI}<18.5,18.5-25,25-30$, or $\geq 30$, respectively), education (high school; less/more than high school), urban code (central or rural area), income (proportion to poverty level) and smoking status, as collected by NHANES. Aspirin use was defined according to previous literature on the same population [28]. PESA and PISA were derived from the available periodontal measures, as explained elsewhere [25]. Additional parameters of interest included serum levels of inflammatory markers (C-reactive protein [CRP], ferritin, white blood cells [WBC]), and lipid panel (low- and high-density lipoprotein cholesterol [LDL-C, HDL-c], triglycerides).

\section{Statistical analysis}


The primary goal of this study was to test the association of bleeding gums with BP. The secondary aims were testing how periodontitis impacts on the observed relation and whether systemic inflammatory markers could predict the association. BP was the dependent variable. In order to test our hypothesis in a controlled setting, we adopted a survey-based propensity score matching (PSM) $[29,30]$ a technique used in observational studies to aid in the evaluation of cause-effect hypotheses $[29,31]$ and to reduce bias in the effect estimates by ensuring balance on the observed variables between groups [29,32]. Specifically, 1:1 nearest-neighbor matching was done by weighted logistic generalized additive model (GAM) for possible confounders in the association between hypertension and periodontal disorders (age, gender, ethnicity, poverty, BMI, hypertension diagnosis and treatment, smoking habit, diabetic status, and aspirin use) $[30,33]$. Since balance between groups was achieved, the covariates used in PSM were excluded from further adjustments due to possible overadjustment [34]. Additional adjustments were made for LDL-c, CRP, ferritin, and WBC.

Fitted systolic BP means and odds ratios (ORs) for high/uncontrolled BP according to gingival bleeding were then obtained from both weighted and unweighted logistic GAM [35,36].

Stratification for periodontal status (healthy, gingivitis, stable periodontitis, unstable periodontitis), age ranges, and antihypertensive treatment status (untreated patients) was also performed.

In order to assess the weight of the single variables included in the adjustments, variables importance analysis [37] was performed using a highly effective machine learning (ML) approach based on extreme gradient boosting using 10-fold cross-validation [38-40]. The model was trained using random sampling of $80 \%$ of total observations, while its prediction accuracy rate was tested on the remaining $20 \%$. Finally, in order to test the impact of gingival bleeding burden on BP, we performed a sensitivity analysis excluding patients with periodontitis from the sample. The remaining participants were then stratified into 3 groups, i.e. healthy (BoP $<10 \%$ ), localized gingivitis (BoP 10\%-30\%), and generalized gingivitis (BoP >30\%) [20].

All statistical analyses were performed using R (v3.6.0) [41] accounting for a complex multistage probability cluster survey design. Differences in clinical and demographic characteristics were 
evaluated with unpaired t-tests for continuous variables and $\chi^{2}$ tests for categorical variables. Bonferroni correction was applied as appropriate. Data were analyzed as recorded, without imputation for missing data. Statistical significance was set at $p<0.05$.

\section{Results}

A total of 5,396 NHANES III participants, selected by PSM and equally distributed in two groups (bleeding gums and non-bleeding gums; $n=2698 /$ group), underwent the analyses of interest. The groups were homogeneous in terms of age, gender, ethnicity, poverty, BMI, hypertension diagnosis, use of antihypertensive medications, smoking habits, and diabetic status ( $p>0.05)$, as expected (Table 1). No difference in terms of estimated glomerular filtration rate (eGFR) and aspirin use were reported between the two groups. Crude systolic BP was $1.5 \mathrm{mmHg}$ higher $(p=0.004)$ in participants with bleeding gums compared with those with non-bleeding gums (Table 1). Multivariate analysis increased this difference to $2.6 \mathrm{mmHg}(\mathrm{p}<0.001)$ (S Table 1). Mean serum levels of inflammatory markers tended to be lower among participants with non-bleeding gums, without reaching statistical significance (Table 1).

Mean adjusted systolic BP progressively increased from healthy patients to those with gingivitis (+2 mmHg, $\mathrm{p}<0.001)$, stable periodontitis $(+5.2 \mathrm{mmHg}, \mathrm{p}<0.001)$, and unstable periodontitis $(+7.3$ $\mathrm{mmHg}, \mathrm{p}<0.001)$ ( $\mathrm{p}<0.001$ for multiple comparisons) (Table 2). Bleeding gums were associated with $38 \%$ higher prevalence of $B P \geq 140 / 90 \mathrm{mmHg}(p=0.002)$ and $42 \%$ higher prevalence of BP $\geq 130 / 80 \mathrm{mmHg}(p<0.001)$ (S Table 2). Weighted multivariate analysis revealed that gingivitis was associated with $29 \%$ and $49 \%$ higher prevalence/odds of high/uncontrolled BP according to European and American guidelines, respectively (S Table 3). With reference to stable periodontitis, the magnitude of association of high/uncontrolled BP was similar between guidelines, but it was only significant according to the US target $(+49 \%, p=0.007$; S Table 3$)$. Unstable periodontitis was associated with about twice the odds of $B P \geq 140 / 90 \mathrm{mmHg}(p<0.001)$, and with $65 \%$ higher prevalence of $\mathrm{BP} \geq 130 / 80 \mathrm{mmHg}(\mathrm{p}<0.001)$ (S Table 3 ). GAM spline showed that BoP has a nearly linear relationship with systolic BP independent of periodontitis (estimated degrees of freedom $=0.94 ; F=1.4 ; p<0.001)($ Figure 1). 
After stratification by age, gingival bleeding was associated with increased mean systolic BP compared to non-bleeding gums by about 2 to $3 \mathrm{mmHg}(\mathrm{p}<0.001)$ across all age ranges (S Table 4). Participants with unstable periodontitis had a worse systolic BP profile than those with gingivitis $(+1.7$ to $+2.5 \mathrm{mmHg}, \mathrm{p}<0.001)$ and stable periodontitis $(+2.8$ to $+3.7 \mathrm{mmHg}, \mathrm{p}<0.001)$, independent of age (S Table 5). Interestingly, no difference in BP was observed between healthy gums and stable periodontitis, while participants with gingivitis had higher systolic BP than those with stable periodontitis across all age ranges (S Table 5).

When analyzing untreated hypertensive patients only, individuals with non-bleeding gums $(\mathrm{N}=59)$ had significantly lower fitted mean systolic BP than those with bleeding gums $(N=56)(130.6 \pm 6.5$ versus $139.5 \pm 8.9 \mathrm{mmHg}$, respectively; $p<0.001$ ). They also displayed greater odds of $B P \geq 130 / 80$ $\mathrm{mmHg}$ (weighted adjusted OR 3.07, 95\% Cl 1.21-7.77, $\mathrm{p}=0.02$ ), but not of $\mathrm{BP} \geq 140 / 90 \mathrm{mmHg}$ (weighted adjusted OR 1.99, 95\% Cl 0.92-4.31, $\mathrm{p}=0.084$ ).

When BoP was examined as a continuous variable, the risk of high/uncontrolled BP increased by about $1 \%$ for unitary increase in BoP, independent of guidelines (OR 1.01, C.I. 95\% 1.01-1.02 ; p $<0.001$ ) (S Figure 1).

\section{Inflammatory markers}

Serum levels of inflammatory markers (CRP, ferritin, WBC) were higher among participants with non-bleeding gums who had high/uncontrolled BP compared to those with normal/controlled BP according to US guidelines (S Figure 2, S Table 6). In the presence of gingival bleeding, this finding was observed only for serum ferritin levels (S Figure 2, S Table 6). With reference to European guidelines, only ferritin levels were different by BP status/control, independent of gingival bleeding (S Figure 2, S Table 6).

Mean WBC were higher $(p=0.008)$ in the presence of bleeding gums compared with no bleeding among participants with $\mathrm{BP}<130 / 80 \mathrm{mmHg}$ (S Figure 2, S Table 7). Conversely, inflammatory markers did not differ by gingival bleeding status among participants with high/uncontrolled BP (S Figure 2, S Table 7). 


\section{$\underline{M L}$ and variables importance}

ML predicted the outcome with an accuracy rate of $70.3 \%$ (sensitivity $=0.79$; specificity $=0.61$ ) when the ACC/AHA guidelines were applied, and of $78.4 \%$ (sensitivity=0.92; specificity=0.37) when the European guidelines were used as reference.

Being diagnosed as not having hypertension accounted for about 30 to $40 \%$ of the gain in the predictive power of the model, followed by older age with over $25 \%$ (S Figure 3, S Table 8). Having bleeding gums enhanced the predictive power of the model by about $0.3-1 \%$. Serum inflammatory mediators allowed a gain in the model's predictive power to a greater extent when applying the European (about 12\%) than the US (about 3.1\%) guidelines (S Figure 3, S Table 8). Notably, bleeding gums enhanced the predictive power of the model more than diabetes when ESC/ESH guidelines were used as reference (1.0\% vs $0.8 \%$, respectively). Moreover, the single contribution of race, smoking habits, and glycolipid parameters was substantially similar to or lower than that of inflammatory markers (S Figure 3, S Table 8).

\section{Sensitivity analysis}

This subset consisted of a total of 3679 NHANES III participants who were free of any form of periodontitis. They were homogeneous in terms of gender, age, glycemic status, smoking habits, prevalence of diagnosed hypertension, use of aspirin, and use of antihypertensive medications. Of them, about $33.5 \%$ had a localized, while $12.5 \%$ a generalized form of gingivitis; the remaining $54 \%$ had healthy gums. Mean systolic BP increased according to the extension of gingivitis ( $p<0.001$ for multiple comparisons). In particular, participants with the localized form shown greater systolic BP compared with healthy individuals $(+1.1 \mathrm{mmHg}, \mathrm{p}<0.001)$, while the generalized form increased systolic BP by $+4.7 \mathrm{mmHg}(\mathrm{p}<0.001)$ after multivariate adjustment (S Table $\mathbf{9})$. Association analysis on 1551 participants indicated an increased risk of $B P \geq 130 / 80 \mathrm{mmHg}$ in the presence of localized (OR 1.32, $\mathrm{p}=0.018$ ) and generalized (OR 2.20, $\mathrm{p}<0.001$ ) gingivitis (S Table 10). Conversely, no significant relation was found for either localized or generalized gingivitis with high/uncontrolled BP based on European guidelines (S Table 10). 


\section{Discussion}

Our findings indicate that gingival bleeding is independently associated with higher mean systolic BP by $2.6 \mathrm{mmHg}$, and with about $40 \%$ greater odds of high/uncontrolled BP compared with non-bleeding. In terms of periodontal health status, gingivitis and unstable periodontitis were consistently associated with higher mean systolic BP and greater odds of high/uncontrolled BP compared with healthy periodontium. The magnitude of the observed effect was greater for unstable periodontitis than gingivitis. Serum ferritin levels were higher in participants with high/uncontrolled BP than in those with normal/controlled BP independent of bleeding status, while no difference in terms of inflammatory mediators was found among participants with high/uncontrolled BP with either bleeding or non-bleeding gums. The available inflammatory markers allowed a maximum of $12 \%$ gain in the predictive power of the model, while gingival bleeding enhanced the model prediction power more than established CV risk factors, like diabetes, overweight/obesity, and smoking, depending on the guidelines used as reference.

These results expand current knowledge of systemic consequences of periodontal pathology, allowing for a better understanding of the impact that periodontal diseases exert on BP. Specifically, it appears that presence of gingival bleeding is relevant in shaping this relationship, but the additional burden represented by periodontitis should not be underestimated. This is in line with our recent observation that acute periodontal inflammation is associated with BP independent of periodontitis parameters, which, however, might further amplify the magnitude of the association [25]. In addition, the observed findings extend previous evidence on the periodontitis-BP relation, where an estimate of disease activity was not available [19]. The unique contribution of specific and diverse inflammatory pathways in the acute and the chronic settings might be a possible explanation for the observed differences between gingivitis and stable/unstable periodontitis in the reported association $[42,43]$. The potential immunological and metabolic effects of fluctuations in local microflora might also exert a pathogenic role in the same relation, as described for other diseases [43-46]. The findings from untreated hypertensive patients confirm the overall results, and add to our previous findings on 11753 participants from NHANES 2009-2014 campaigns, where bleeding on probing was not available. In fact, the difference in mean BP (about $9 \mathrm{mmHg}$ ) 
was substantially greater than that observed in the overall results $(2.6 \mathrm{mmHg})$, suggesting that antihypertensive treatment reduces, but does not offset, this difference between groups of periodontal status. A possible explanation for the non-significant increase in the risk of high/uncontrolled BP according to the European guidelines is that, given the small number of patients, the more conservative threshold for high $\mathrm{BP}(130 / 80 \mathrm{mmHg})$ allows to outline betweengroups differences better than the higher cutoff $(140 / 90 \mathrm{mmHg})$.

The observation of similar levels of serum inflammatory markers among participants with high/uncontrolled BP independent of gingival bleeding status in our analysis might be the expression of a pro-inflammatory environment, a condition that has been described independently for both hypertension and periodontal disorders. WBC, a routinely available measure of systemic inflammation, are in fact known to be involved in vascular damage and atherosclerosis [47-50], and have been indicated as predictors of hypertension and adverse CV outcomes $[51,52]$. Both lymphocytes [53] and neutrophils [54] appear to mediate these associations. It is now established that $\mathrm{T}$ cells are central in the development of hypertension and related vascular abnormalities, effects that were at least in part mediated by the release of reactive oxygen species (ROS), tumor necrosis factor (TNF) alpha, and interferon (IFN) gamma [49]. In addition, elevated neutrophil-tolymphocyte ratio, a novel marker of inflammation and oxidative stress, has been associated with incident and uncontrolled hypertension [51], as well as with increased mortality among hypertensive individuals [55]. In the same manner, the immune response has an established pivotal role in the onset and progression of periodontitis [56,57]. As an example, Dutzan et al. demonstrated that dysbiosis-dependent $T_{H} 17$ cells expansion, and associated neutrophil accumulation, drive human periodontal disease, and that people who are naturally deficient in $T_{H} 17$ cells were less likely to develop periodontitis [42]. Interestingly, a central role of $T_{H} 17$ cells has also been described in hypertension, where they are involved in multiple pathogenetic events, from sodium/water retention, to increased ROS production, decreased nitric oxide (NO) bioavailability, and fibrosis, thus contributing to hypertension-mediated organ damage $[58,59]$. Interleukin (IL)-1 $\beta$, IL-8, IL-6, and TNF alpha derived from activated neutrophils after stimulation by periodontal pathogens are abundant in periodontitis patients [56], and are also constantly elevated in 
hypertension [60]. Observational evidence from a large population-based study indicated higher levels of CRP and fibrinogen among participants who never or rarely brushed their teeth [61].

In parallel, bleeding gums are a recognized keystone sign of oral inflammation with systemic impact $[20,62,63]$. There is evidence of a strong association between BoP and serum biomarkers of inflammation in populations with different CV risk [64,65]. Increased BoP has been also associated with a hyper-inflammatory phenotype related to allelic variants of IL-1 gene [66]. Interestingly, self-reported bleeding gums have been associated with a $77 \%$ higher risk of hypertension [67]. As clinical BoP is associated with self-reported gums bleeding [20,68,69], the clinician faced with the management of hypertension could easily identify individuals at increased risk for high/uncontrolled BP by simply adding a question on gingival bleeding status when collecting a patient's medical history (i.e. "Do your gums bleed during tooth brushing?"). With periodontitis now acknowledged as a modifiable non-traditional risk factor for CVD, the potential clinical implications of this approach should not be discarded. As reported in the latest PerioCardio workshop promoted by the European Federation of Periodontology (EFP) and the World Heart Federation (WHF), in fact, robust epidemiological evidence has accumulated in the past decades in support of an association between periodontal disorders and CVD [56]. Further, findings from recent genetic studies have indicated a causal relationship between periodontitis and clinical/subclinical CVD. In particular, genetic variants in CDKN2B-AS1 (ANRIL), PLG, CAMTA1/VAMP3, and VAMP8 loci were described to determine an aberrant inflammatory phenotype that could partially explain the epidemiological link between periodontitis and CVD [56]. On this ground, the Perio-Cardio Consensus Report recommended oral and periodontal examination in patients with CVD, and warned from the increased CV risk in the presence of periodontitis [56]. Whether the same approach in patients with hypertension would translate into a similar cost-effective measure is yet to be determined; however, our results add to the existing observational evidence in support of this possibility. This is in line with the conclusions of a recent meta-analysis on the association of periodontitis and hypertension [17]. A recent randomized controlled trial reporting on the benefit on BP of non-surgical periodontal therapy, combined with a Mendelian Randomization analysis demonstrating a significant relationship between periodontitis- 
linked gene variants and BP phenotypes, provided the first evidence of a causal association between periodontitis and hypertension [18]. Oral health promotion is not only based on professionally rendered oral care measures. Indeed tooth brushing is a widely available, safe and low-cost option to preserve good oral health and reduce the oral inflammatory burden $[70,71]$. The American Dental Association and EFP recommend properly performed tooth brushing twice daily $[72,73]$. The benefits of this practice appear to go beyond the boundaries of the oral cavity. In fact, a survey of 19,560 adults indicated that the prevalence of high/uncontrolled BP decreased as the frequency of tooth-brushing increased, independent of periodontitis [74], confirming this oral health measure as the most important aid against gingivitis [20]. In addition, tooth-brushing at least one per day was associated with a $9 \%$ lower risk of CV events after 9.5 years of follow-up [75]. The same practice three times a day or more compared with once or less per day was associated with a $19 \%$ reduction in CV events, while regular professional cleaning reduced the same risk by $14 \%$ [75]. Thus, a simple question regarding bleeding gums might represent a reliable and easily accessible tool in daily clinical practice to assess periodontal inflammation and help in easily identifying patients at greater risk for hypertension. A multidisciplinary approach for the management of hypertension should involve oral care experts in a not too distant future with the aim of contributing to the reduction of total CVD risk.

This study is not without limitations. First, since an analysis of observational, crosssectional data was performed, a causality or temporal relationship between the analyzed variables cannot be inferred, and dedicated trials are needed to test the effect of periodontal treatment on hypertension, as well as to exclude a possible reverse causation. However, evidence of hypertension-mediated microvessels damage at the periodontal level is not available to date. The exclusion of institutionalized patients from the survey might have determined a certain degree of selection bias. According to NHANES III protocol, periodontal indices were assessed only in 2 randomly selected quadrants in the enrolled patients, with consequent possible underestimation of periodontitis [76]. In addition, the potential impact of unmeasured clinical features with effect on BP or oral health, including alcohol use $[77,78]$, or disparities in socioeconomic status determining different degrees of oral health awareness or health care access, should be also considered. More 
recent survey campaigns have been performed, but BoP was only available in NHANES III. Finally, due to the lack of information on hypertension drug classes, number and dose of medications, appropriateness of antihypertensive agents, use of anticoagulants, and adherence to therapy, the potential impact of each of these factors on the observed findings could not be tested. It must be noted, however, that although some calcium channel blockers, like nifedipine and amlodipine, have been associated with gingival overgrowth [79], they do not appear to induce gingival bleeding [80].

However, this study also has several strengths. PSM allowed controlling for major confounders in the relation between periodontal disorders and BP, and further adjustment was made for relevant variables. The impact of gingival bleeding on hypertension was assessed across the clinical spectrum of periodontal diseases in participants of a large, multiethnic, nationally representative survey. Stratification by severity of gingivitis allowed an estimate of the effect magnitude. Variables importance in the examined association was assessed using a highly effective $M L$ technique.

In conclusion, an easily accessible marker of periodontal health status as gingival bleeding contributes to shaping the relationship between periodontal diseases and BP, but the burden represented by periodontitis is remarkable. In the light of the recently acknowledged role of periodontal disorders as a modifiable CV risk factor [56,81], our findings indicate a rationale for considering a good periodontal health as a prevention strategy also for hypertension. Oral and periodontal evaluation might be of importance not only in established CVD, but also in hypertensive patients, especially in cases where gingival bleeding is detected. 


\section{Acknowledgements}

We would like to acknowledge the Department Chair Prof. Guido Macchiarelli and the Rector of the University of L'Aquila Prof. Edoardo Alesse who provided general support.

\section{Funding}

The authors received no financial support for the research, authorship, and/or publication of this article. EMA and FD work at UCLH/UCL who received a proportion of funding from the Department of Health's NIHR Biomedical Research Centre funding scheme. 


\section{Conflict of Interest}

All the Authors declare no conflict of interest

\section{Authors' Contributions}

DP and RDP contributed equally to this paper. DP and RDP contributed to the conception and the design of the work, acquisition and data analysis. DP, RDP, FDA, EMA, CF, AM, EO, MG, MCG and TG contributed to interpretation of data for the work. DP, RDP, EO and EMA drafted the manuscript. AM, MG, FDA, MCG, TG and CF critically revised the manuscript.

All gave final approval and agree to be accountable for all aspects of work ensuring integrity and accuracy. 


\section{References}

1 Piepoli MF, Hoes AW, Agewall S, Albus C, Brotons C, Catapano AL, et al. 2016 European Guidelines on cardiovascular disease prevention in clinical practice: The Sixth Joint Task Force of the European Society of Cardiology and Other Societies on Cardiovascular Disease Prevention in Clinical Practice (constituted by representatives of 10 societies and by invited experts)Developed with the special contribution of the European Association for Cardiovascular Prevention \& Rehabilitation (EACPR). Eur Heart J 2016; 37:2315-2381.

2 Forouzanfar MH, Liu P, Roth GA, Ng M, Biryukov S, Marczak L, et al. Global Burden of Hypertension and Systolic Blood Pressure of at Least 110 to $115 \mathrm{~mm} \mathrm{Hg}, 1990-2015$. JAMA $2017 ; 317: 165-182$.

3 Banegas JR, Lopez-Garcia E, Dallongeville J, Guallar E, Halcox JP, Borghi C, et al. Achievement of treatment goals for primary prevention of cardiovascular disease in clinical practice across Europe: the EURIKA study. European Heart Journal. 2011; 32:2143-2152.

4 Del Pinto R, Pagliacci S, De Feo M, Grassi D, Ferri C, Italian Society of Hypertension and Federfarma. Prevalence of hypertension and associated cardiovascular risk factors among pharmacies customers: an Italian nationwide epidemiological survey. Eur J Prev Cardiol 2019; :2047487319851301.

5 Williams B, Mancia G, Spiering W, Agabiti Rosei E, Azizi M, Burnier M, et al. 2018 ESC/ESH Guidelines for the management of arterial hypertension: The Task Force for the management of arterial hypertension of the European Society of Cardiology and the European Society of Hypertension: The Task Force for the management of arterial hypertension of the European Society of Cardiology and the European Society of Hypertension. J Hypertens 2018; 36:1953-2041.

6 Loos BG, Craandijk J, Hoek FJ, Wertheim-van Dillen PM, van der Velden U. Elevation of systemic markers related to cardiovascular diseases in the peripheral blood of periodontitis 
patients. J Periodontol 2000; 71:1528-1534.

7 Winning L, Linden GJ. Periodontitis and systemic disease. BDJ Team 2015; 2:567.

8 Kassebaum NJ, Bernabé E, Dahiya M, Bhandari B, Murray CJL, Marcenes W. Global burden of severe periodontitis in 1990-2010: a systematic review and meta-regression. J Dent Res 2014; 93:1045-1053.

9 Kinane D, Bouchard P, Group E of European Workshop on Periodontology. Periodontal diseases and health: Consensus Report of the Sixth European Workshop on Periodontology. J Clin Periodontol 2008; 35:333-337. Periodontal health and gingival diseases and conditions on an intact and a reduced periodontium: Consensus report of workgroup 1 of the 2017 World Workshop on the Classification of Periodontal and Peri-Implant Diseases and Conditions. J Periodontol 2018; 89 Suppl 1:S74-S84.

11 Nesse W, Abbas F, van der Ploeg I, Spijkervet FKL, Dijkstra PU, Vissink A. Periodontal inflamed surface area: quantifying inflammatory burden. J Clin Periodontol 2008; 35:668-673. Tonetti MS, Van Dyke TE, working group 1 of the joint EFP/AAP workshop. Periodontitis and atherosclerotic cardiovascular disease: consensus report of the Joint EFP/AAP Workshop on Periodontitis and Systemic Diseases. J Periodontol 2013; 84:S24-9. Severity of periodontal disease correlates to inflammatory systemic status and independently predicts the presence and angiographic extent of stable coronary artery disease. $J$ Intern Med 2008; 263:644-652. 
al. Randomized controlled trial of the effect of periodontal treatment on cardiovascular risk biomarkers in patients with stable coronary artery disease: Preliminary findings of 3 months. $J$ Clin Periodontol 2019; 46:321-331.

15 Tonetti MS, Greenwell H, Kornman KS. Staging and grading of periodontitis:

Framework and proposal of a new classification and case definition. Journal of Periodontology. 2018; 89:S159-S172. Published Online First: 8 January 2020. doi:10.1111/jcpe.13253

17 Muñoz Aguilera E, Suvan J, Buti J, Czesnikiewicz-Guzik M, Barbosa Ribeiro A, Orlandi $\mathrm{M}$, et al. Periodontitis is associated with hypertension: a systematic review and metaanalysis. Cardiovasc Res Published Online First: 24 September 2019. doi:10.1093/cvr/cvz201

18 Czesnikiewicz-Guzik M, Osmenda G, Siedlinski M, Nosalski R, Pelka P, Nowakowski D, et al. Causal association between periodontitis and hypertension: evidence from Mendelian randomization and a randomized controlled trial of non-surgical periodontal therapy. Eur Heart J 2019; 40:3459-3470.

Oral Health and Blood Pressure Control Among US Hypertensive Adults: Results From the National Health and Nutrition Examination Survey 2009 to 2014. Hypertension 2018; 72:1365-1373.

20 Trombelli L, Farina R, Silva CO, Tatakis DN. Plaque-induced gingivitis: Case definition and diagnostic considerations. J Periodontol 2018; 89 Suppl 1:S46-S73.

21 Ezzati TM, Massey JT, Waksberg J, Chu A, Maurer KR. Sample design: Third National Health and Nutrition Examination Survey. Vital Health Stat $21992 ;$ :1-35. 
Update on Prevalence of Periodontitis in Adults in the United States: NHANES 2009 to 2012. J Periodontol 2015; 86:611-622.

23 Eke PI, Page RC, Wei L, Thornton-Evans G, Genco RJ. Update of the case definitions for population-based surveillance of periodontitis. J Periodontol 2012; 83:1449_ 1454.

24 Westat, Inc. 1650 Research Boulevard Rockville. NATIONAL HEALTH AND NUTRITION EXAMINATION SURVEY III ORAL EXAMINATION COMPONENT. cdc.gov. Revised March 1992.https://wwwn.cdc.gov/nchs/data/nhanes3/manuals/dental.pdf (accessed 2 Dec2019).

25 Pietropaoli D, Del Pinto R, Ferri C, Marzo G, Giannoni M, Ortu E, et al. Association between periodontal inflammation and hypertension using periodontal inflamed surface area and bleeding on probing. J Clin Periodontol Published Online First: 4 November 2019. doi:10.1111/jcpe.13216

26 Tsakos G, Sabbah W, Hingorani AD, Netuveli G, Donos N, Watt RG, et al. Is periodontal inflammation associated with raised blood pressure? Evidence from a National US survey. J Hypertens 2010; 28:2386-2393.

27 Whelton PK, Carey RM, Aronow WS, Casey DE Jr, Collins KJ, Dennison Himmelfarb C, et al. 2017 ACC/AHA/AAPA/ABC/ACPM/AGS/APhA/ASH/ASPC/NMA/PCNA Guideline for the Prevention, Detection, Evaluation, and Management of High Blood Pressure in Adults: A Report of the American College of Cardiology/American Heart Association Task Force on Clinical Practice Guidelines. J Am Coll Cardiol 2018; 71:e127-e248. diabetes: estimates from the Third National Health and Nutrition Examination Survey. Diabetes Care 2001; 24:197-201. 
Comparison of Propensity Score Methods and Covariate Adjustment: Evaluation in 4 Cardiovascular Studies. J Am Coll Cardiol 2017; 69:345-357.

30 Dugoff EH, Schuler M, Stuart EA. Generalizing observational study results: applying propensity score methods to complex surveys. Health Serv Res 2014; 49:284-303.

$31 \quad$ Williamson EJ, Forbes A. Introduction to propensity scores. Respirology 2014; 19:625-635.

32 Austin PC, Jembere N, Chiu M. Propensity score matching and complex surveys. Stat Methods Med Res 2018; 27:1240-1257.

33 Ho DE, Imai K, King G, Stuart EA. Matchlt: Nonparametric Preprocessing for Parametric Causal Inference. Journal of Statistical Software. 2011; 42. doi:10.18637/jss.v042.i08

34 Nguyen T-L, Collins GS, Spence J, Daurès J-P, Devereaux PJ, Landais $\mathrm{P}$, et al. Double-adjustment in propensity score matching analysis: choosing a threshold for considering residual imbalance. BMC Med Res Methodol 2017; 17:78.

35 Yiu S, Su L. Covariate association eliminating weights: a unified weighting framework for causal effect estimation. Biometrika 2018; 105:709-722.

36 Bollen KA, Biemer PP, Karr AF, Tueller S, Berzofsky ME. Are Survey Weights Needed? A Review of Diagnostic Tests in Regression Analysis. Annu Rev Stat Appl 2016; 3:375-392.

37 Wei P, Lu Z, Song J. Variable importance analysis: A comprehensive review. Reliab Eng Syst Saf 2015; 142:399-432.

38 Chen T, Guestrin C. XGBoost: A Scalable Tree Boosting System. In: Proceedings of the 22nd ACM SIGKDD International Conference on Knowledge Discovery and Data Mining - KDD '16.New York, New York, USA: ACM Press; 2016. pp. 785-794. 

2008; 28. doi:10.18637/jss.v028.i05

40 Chen T, He T, Benesty M, Khotilovich V. Extreme Gradient Boosting - Package "xgboost." CRAN. https://cran.r-project.org/web/packages/xgboost/xgboost.pdf (accessed 10 Apr2020).

41 Tippmann S. Programming tools: Adventures with R. Nature 2015; 517:109-110.

42 Dutzan N, Kajikawa T, Abusleme L, Greenwell-Wild T, Zuazo CE, Ikeuchi T, et al. A dysbiotic microbiome triggers $\mathrm{TH} 17$ cells to mediate oral mucosal immunopathology in mice and humans. Sci Transl Med 2018; 10. doi:10.1126/scitranslmed.aat0797

43 Hajishengallis G. Periodontitis: from microbial immune subversion to systemic inflammation. Nat Rev Immunol 2014; 15:30-44.

44 Lamont RJ, Koo H, Hajishengallis G. The oral microbiota: dynamic communities and host interactions. Nat Rev Microbiol 2018; 16:745-759.

45 Del Pinto R, Wright JT, Monaco A, Pietropaoli D, Ferri C. Vitamin D and blood pressure control among hypertensive adults: results from NHANES 2001-2014. J Hypertens 2020; 38:150-158.

46 Del Pinto R, Ferri C, Cominelli F. Vitamin D Axis in Inflammatory Bowel Diseases: Role, Current Uses and Future Perspectives. Int J Mol Sci 2017; 18:2360.

47 Jain S, Khera R, Corrales-Medina VF, Townsend RR, Chirinos JA. "Inflammation and arterial stiffness in humans." Atherosclerosis 2014; 237:381-390.

48 Karino S, Willcox BJ, Fong K, Lo S, Abbott R, Masaki KH. Total and differential white blood cell counts predict eight-year incident coronary heart disease in elderly JapaneseAmerican men: the Honolulu Heart Program. Atherosclerosis 2015; 238:153-158. 

T cell in the genesis of angiotensin II-induced hypertension and vascular dysfunction. The Journal of Experimental Medicine. 2007; 204:2449-2460.

50 Drummond GR, Vinh A, Guzik TJ, Sobey CG. Immune mechanisms of hypertension. Nat Rev Immunol 2019; 19:517-532.

51 Jhuang Y-H, Kao T-W, Peng T-C, Chen W-L, Li Y-W, Chang P-K, et al. Neutrophil to lymphocyte ratio as predictor for incident hypertension: a 9-year cohort study in Taiwan. Hypertens Res 2019; 42:1209-1214.

52 Chen, Chen C, Zhao HY, Zhang YH. Correlation between neutrophil-to-lymphocyte ratio and kidney dysfunction in undiagnosed hypertensive population from general health checkup. The Journal of Clinical Hypertension. 2020; 22:47-56.

53 Andreadou I, Cabrera-Fuentes HA, Devaux Y, Frangogiannis NG, Frantz S, Guzik T, et al. Immune cells as targets for cardioprotection: new players and novel therapeutic opportunities. Cardiovasc Res 2019; 115:1117-1130.

54 Bonaventura A, Montecucco F, Dallegri F, Carbone F, Lüscher TF, Camici GG, et al. Novel findings in neutrophil biology and their impact on cardiovascular disease. Cardiovasc Res 2019; 115:1266-1285.

55 Sun X, Luo L, Zhao X, Ye P, Du R. The neutrophil-to-lymphocyte ratio on admission is a good predictor for all-cause mortality in hypertensive patients over 80 years of age. BMC Cardiovasc Disord 2017; 17:167.

Sanz M, Marco Del Castillo A, Jepsen S, Gonzalez-Juanatey JR, D’Aiuto F, Bouchard P, et al. Periodontitis and cardiovascular diseases: Consensus report. J Clin Periodontol Published Online First: 3 February 2020. doi:10.1111/jcpe.13189 Van Dyke TE, Sima C. Understanding resolution of inflammation in periodontal 
diseases: Is chronic inflammatory periodontitis a failure to resolve? Periodontol 2000 2020; $82: 205-213$.

58 Norlander AE, Madhur MS, Harrison DG. The immunology of hypertension. J Exp Med 2018; 215:21-33.

59 Mikolajczyk TP, Guzik TJ. Adaptive Immunity in Hypertension. Curr Hypertens Rep $2019 ; 21: 68$.

60 Garcia-Fernandez N, Beaumont J, Moreno MU, San José G, González A, Díez J. The renal immune-inflammatory component of arterial hypertension: emerging therapeutic strategies. Cardiovasc. Res. 2019; 115:696-698.

61 de Oliveira C, Watt R, Hamer M. Toothbrushing, inflammation, and risk of cardiovascular disease: results from Scottish Health Survey. BMJ 2010; 340:c2451.

62 Mysak J, Podzimek S, Vasakova J, Mazanek J, Vinsu A, Duskova J. C-reactive protein in patients with aggressive periodontitis. J Dent Sci 2017; 12:368-374.

63 Eberhard J, Grote K, Luchtefeld M, Heuer W, Schuett H, Divchev D, et al. Experimental gingivitis induces systemic inflammatory markers in young healthy individuals: a single-subject interventional study. PLoS One 2013; 8:e55265.

64 Bokhari SAH, Khan AA, Butt AK, Hanif M, Izhar M, Tatakis DN, et al. Periodontitis in coronary heart disease patients: strong association between bleeding on probing and systemic biomarkers. J Clin Periodontol 2014; 41:1048-1054.

65 Beck JD, Offenbacher S. Relationships among clinical measures of periodontal disease and their associations with systemic markers. Ann Periodontol 2002; 7:79-89.

66 Lang NP, Tonetti MS, Suter J, Sorrell J, Duff GW, Kornman KS. Effect of interleukin1 gene polymorphisms on gingival inflammation assessed by bleeding on probing in a periodontal maintenance population. J Periodontal Res 2000; 35:102-107. 
67 Buhlin K, Gustafsson A, Håkansson J, Klinge B. Oral health and cardiovascular disease in Sweden. J Clin Periodontol 2002; 29:254-259.

68 Kallio P, Nordblad A, Croucher R, Ainamo J. Self-reported gingivitis and bleeding gums among adolescents in Helsinki. Community Dent Oral Epidemiol 1994; 22:277-282.

69 Kallio P. Self-assessed bleeding in monitoring gingival health among adolescents. Community Dent Oral Epidemiol 1996; 24:128-132.

70 Hettiarachchi RM, Kularatna S, Downes MJ, Byrnes J, Kroon J, Lalloo R, et al. The cost-effectiveness of oral health interventions: A systematic review of cost-utility analyses. Community Dent Oral Epidemiol 2018; 46:118-124.

71 Barone A, Giannoni M, Ortu E, Monaco A, Pietropaoli D. Short-term and Longlasting Effects of Hypo-Cariogenic Dietary Advice and Oral Care on Oral Flora: a Randomised Clinical Trial. Oral Health Prev Dent 2018; 16:315-325.

72 Home Oral Care. https://www.ada.org/en/member-center/oral-health-topics/homecare (accessed 10 Apr2020). West N, Jepsen S, Chapple I, Tonetti M. TOOTH DECAY AND GUM DISEASE Recommendations for the public. European Federation of Periodontology. 2016.https://www.efp.org/publications/projects/perioandcaries/recommendations/Guideline04 _Public.pdf (accessed 10 Apr2020).

74 Choi HM, Han K, Park Y-G, Park J-B. Associations Among Oral Hygiene Behavior and Hypertension Prevalence and Control: The 2008 to 2010 Korea National Health and Nutrition Examination Survey. J Periodontol 2015; 86:866-873. hygiene care attenuates the cardiovascular risk of oral health disease: a population-based study from Korea. Eur Heart J 2019; 40:1138-1145. 
76 Eke PI, Thornton-Evans GO, Wei L, Borgnakke WS, Dye BA. Accuracy of NHANES periodontal examination protocols. J Dent Res 2010; 89:1208-1213.

77 Del Pinto R, Pietropaoli D, Ferri C. Diastolic blood pressure and risk profile in renal and cardiovascular diseases. Results from the SPRINT trial. J Am Soc Hypertens 2018; 12:513-523.e3.

78 Townsend RR, Chang TI, Cohen DL, Cushman WC, Evans GW, Glasser SP, et al. Orthostatic changes in systolic blood pressure among SPRINT participants at baseline. J Am Soc Hypertens 2016; 10:847-856.

79 Vidal F, de Souza RC, Ferreira DC, Fischer RG, Gonçalves LS. Influence of 3 calcium channel blockers on gingival overgrowth in a population of severe refractory hypertensive patients. J Periodontal Res 2018; 53:721-726.

80 Hughes FJ, Bartold PM. Periodontal complications of prescription and recreational drugs. Periodontol 2000 2018; 78:47-58.

81 Cho D-H, Song I-S, Choi J, Gwon JG. Risk of peripheral arterial disease in patients with periodontitis: A nationwide, population-based, matched cohort study. Atherosclerosis Published Online First: February 2020. doi:10.1016/j.atherosclerosis.2020.02.012 


\section{Figure legends}

Figure 1. Full adjusted GAM spline of the relationship between the continuum of BoP (\%) and systolic BP $(\mathbf{m m H g})$. Patients with periodontitis showed constantly higher systolic BP (red) compared to those without the disease (blue). The difference in systolic BP between the two groups appears constant across the continuum of BoP, resulting in an almost linear relationship. 


\section{Tables}

Table 1. Clinical and demographic characteristics of NHANES III participants selected by propensity score matching. Groups were homogeneous for possible confounders in the association between hypertension and periodontal diseases (gender, age, ethnicity, race, BMI, diabetes, poverty, smoking, hypertension diagnosis and medications, aspirin use).

\begin{tabular}{|c|c|c|c|c|}
\hline Strata & Levels & $\begin{array}{c}\text { Non-bleeding } \\
\text { gums } \\
(\mathrm{N}=2698)\end{array}$ & $\begin{array}{l}\text { Bleeding } \\
\text { gums } \\
(\mathrm{N}=2698)\end{array}$ & $P$ value \\
\hline Gender (\%) & Women & $1281(47.5)$ & $1255(46.5)$ & 0.495 \\
\hline \multirow[t]{3}{*}{ Age, years (\%) } & $<45$ & $1351(50.1)$ & $1325(49.1)$ & 0.665 \\
\hline & $45-65$ & $846(31.4)$ & $848(31.4)$ & \\
\hline & $\geq 65$ & $501(18.6)$ & 525 (19.5) & \\
\hline Ethnicity (\%) & Hispanic & $983(36.4)$ & $1011(37.5)$ & 0.446 \\
\hline \multirow[t]{4}{*}{ Race (\%) } & Non-Hispanic White & 897 (33.2) & $906(33.6)$ & 0.264 \\
\hline & Non-Hispanic Black & $793(29.4)$ & 734 (27.2) & \\
\hline & Mexican-American & 896 (33.2) & 950 (35.2) & \\
\hline & Other & $112(4.2)$ & $108(4.0)$ & \\
\hline \multirow[t]{4}{*}{ BMI (\%) } & Underweight & $30(1.1)$ & $28(1.0)$ & 0.879 \\
\hline & Normal & $830(30.8)$ & $811(30.1)$ & \\
\hline & Overweight & $1029(38.1)$ & 1025 (38.0) & \\
\hline & Obese & $809(30.0)$ & 834 (30.9) & \\
\hline \multirow[t]{3}{*}{ Diabetic status (\%) } & Normal & $1958(72.6)$ & $1931(71.6)$ & 0.446 \\
\hline & Pre-diabetes & $479(17.8)$ & $478(17.7)$ & \\
\hline & Diabetes & $261(9.7)$ & $289(10.7)$ & \\
\hline
\end{tabular}




\begin{tabular}{|c|c|c|c|c|}
\hline $\begin{array}{r}\text { Poverty (mean } \\
(\mathrm{SD}))\end{array}$ & & $2.22(1.56)$ & $2.18(1.64)$ & 0.405 \\
\hline Smokers (\%) & Yes & $1305(48.4)$ & $1284(47.6)$ & 0.586 \\
\hline \multirow[t]{4}{*}{ US region (\%) } & Northeast & $318(11.8)$ & $308(11.4)$ & 0.626 \\
\hline & Midwest & 477 (17.7) & $445(16.5)$ & \\
\hline & South & $1214(45.0)$ & $1245(46.1)$ & \\
\hline & West & $689(25.5)$ & $700(25.9)$ & \\
\hline Urban code (\%) & $\begin{array}{r}\text { Central counties } \\
>1 \mathrm{MLN}\end{array}$ & $1326(49.1)$ & 1345 (49.9) & 0.624 \\
\hline Taking aspirin (\%) & Yes & $266(9.9)$ & $234(8.7)$ & 0.147 \\
\hline HTN diagnosis (\%) & Yes & $801(29.7)$ & $799(29.6)$ & 0.976 \\
\hline $\begin{array}{r}\text { Taking HTN meds } \\
(\%)\end{array}$ & Yes & $464(77.1)$ & $443(74.5)$ & 0.321 \\
\hline $\begin{array}{r}\text { High/uncontrolled } \\
\text { BP }(2017 \\
\text { ACC/AHA) }(\%)\end{array}$ & $\geq 13080 \mathrm{mmHg}$ & $1389(51.6)$ & $1432(53.2)$ & 0.270 \\
\hline $\begin{array}{c}\text { High/uncontrolled } \\
\text { BP }(2018 \\
\text { ESC/ESH) (\%) }\end{array}$ & $\geq 14090 \mathrm{mmHg}$ & $663(24.6)$ & $732(27.2)$ & 0.037 \\
\hline $\begin{array}{r}\mathrm{SBP}, \mathrm{mmHg}(\text { mean } \\
(\mathrm{SD}))\end{array}$ & & $126.6(18.5)$ & $128.1(19.3)$ & 0.004 \\
\hline $\begin{array}{r}\mathrm{DBP}, \mathrm{mmHg}(\text { mean } \\
(\mathrm{SD}))\end{array}$ & & $76.4(10.1)$ & $77.1(10.5)$ & 0.021 \\
\hline $\begin{array}{r}\text { eGFR, } \mathrm{mL} / \mathrm{min} / 1.73 \\
\mathrm{~m}^{2}(\operatorname{mean}(\mathrm{SD}))\end{array}$ & & $76.60(17.92)$ & 76.64 (18.34) & 0.942 \\
\hline Periodontitis (\%) & Yes & 709 (26.3) & $1008(37.4)$ & $<0.001$ \\
\hline \multirow[t]{3}{*}{$\begin{array}{r}\text { Periodontal status } \\
(\%)\end{array}$} & Healthy & 1989 (73.7) & $0(0.0)$ & $<0.001$ \\
\hline & Gingivitis & $0(0.0)$ & $1690(62.6)$ & \\
\hline & Stable periodontitis & 709 (26.3) & $0(0.0)$ & \\
\hline
\end{tabular}




\begin{tabular}{|c|c|c|c|c|}
\hline & Unstable periodontitis & $0(0.0)$ & $1008(37.4)$ & \\
\hline $\begin{array}{r}\text { PESA, } \mathrm{mm}^{2} \text { (mean } \\
\text { (SD)) }\end{array}$ & & $329.78(124.06)$ & $\begin{array}{c}382.25 \\
(157.16)\end{array}$ & $<0.001$ \\
\hline $\begin{array}{r}\text { PISA, } \mathrm{mm}^{2} \text { (mean } \\
\text { (SD)) }\end{array}$ & & $8.88(15.59)$ & $111.79(97.49)$ & $<0.001$ \\
\hline BoP (mean (SD)) & & $2.19(3.07)$ & 26.95 (16.99) & $<0.001$ \\
\hline \multirow[t]{3}{*}{ BoP status (\%) } & $\mathrm{BoP}=0 \%$ & $1685(62.5)$ & $0(0.0)$ & $<0.001$ \\
\hline & BoP $<10 \%$ & $1013(37.5)$ & $0(0.0)$ & \\
\hline & $\mathrm{BoP} \geq 10 \%$ & $0(0.0)$ & 2698 (100.0) & \\
\hline \multirow[t]{6}{*}{$\begin{array}{r}\text { Self-reported health } \\
\text { status (\%) }\end{array}$} & Excellent & $1010(37.4)$ & 815 (30.2) & $<0.001$ \\
\hline & Very good & $720(26.7)$ & $666(24.7)$ & \\
\hline & Good & $688(25.5)$ & $878(32.5)$ & \\
\hline & Fair & $182(6.7)$ & $222(8.2)$ & \\
\hline & Poor & $17(0.6)$ & $35(1.3)$ & \\
\hline & Not applicable & $81(3.0)$ & $82(3.0)$ & \\
\hline $\begin{array}{r}\text { WBC, } 10^{9} \text { count } / \mathrm{L} \\
(\text { mean }(\mathrm{SD}))\end{array}$ & & $7.14(2.47)$ & $7.25(2.29)$ & 0.090 \\
\hline $\begin{array}{r}\text { Ferritin, ng/mL } \\
(\text { mean (SD)) }\end{array}$ & & $149.18(158.76)$ & $\begin{array}{c}151.54 \\
(170.83)\end{array}$ & 0.602 \\
\hline $\begin{array}{r}\text { CRP, g/dL (mean } \\
(S D))\end{array}$ & & $0.48(0.81)$ & $0.51(0.96)$ & 0.122 \\
\hline $\begin{array}{r}\mathrm{LDL}, \mathrm{mg} / \mathrm{dL} \text { (mean } \\
(\mathrm{SD}))\end{array}$ & & $129.97(37.69)$ & $131.41(37.37)$ & 0.359 \\
\hline
\end{tabular}




\begin{tabular}{|c|c|c|c|c|}
\hline $\begin{array}{r}\mathrm{HDL}, \mathrm{mg} / \mathrm{dL}(\text { mean } \\
(\mathrm{SD}))\end{array}$ & & $50.14(15.38)$ & $50.10(16.51)$ & 0.920 \\
\hline $\begin{array}{r}\text { Triglycerides, } \\
\mathrm{mg} / \mathrm{dL}(\text { mean }(S D))\end{array}$ & & $152.83(143.91)$ & $\begin{array}{c}161.45 \\
(183.47)\end{array}$ & 0.099 \\
\hline $\begin{array}{r}\text { NHANES III } \\
\text { campaign (\%) }\end{array}$ & $1991-1994$ & $1539(57.0)$ & $1234(45.7)$ & $<0.001$ \\
\hline
\end{tabular}

Table footnote:

SD: standard deviation; BMI: body mass index; HTN: hypertension; BP: blood pressure; ACC/AHA: American College of Cardiology/American Heart Association; ESC/ESH: European Society of Cardiology/European Society of Hypertension; SBP: systolic blood pressure; DBP: diastolic blood pressure; eGFR: estimated glomerular filtration rate; PESA: periodontal epithelial surface area; PISA: periodontal inflamed surface area; BoP: bleeding on probing; WBC: white blood cells; CRP: C-reactive protein; LDL: Low-density lipoprotein; HDL: high-density lipoprotein; NHANES: National Health and Nutrition Examination Survey. 
Table 2. Multivariate fitted SBP according to periodontal status. Mean difference in SBP $(\triangle \mathrm{SBP})$ by strata of periodontal status is presented.

\begin{tabular}{|c|c|c|c|c|c|c|}
\hline \multirow{2}{*}{$\begin{array}{l}\text { Weighting } \\
\text { method }\end{array}$} & \multirow[t]{2}{*}{ Periodontal status } & \multirow[t]{2}{*}{$\mathbf{N}$} & \multirow{2}{*}{$\begin{array}{c}\text { SBP } \\
\text { mean (SD) }\end{array}$} & \multicolumn{3}{|c|}{ Multiple comparisons $\Delta$ SBP (P-value) } \\
\hline & & & & Healthy & Gingivitis & $\begin{array}{c}\text { Stable } \\
\text { periodontitis }\end{array}$ \\
\hline \multirow[t]{4}{*}{ Weighted } & Healthy & 840 & $121.3(4.7)$ & - & & \\
\hline & Gingivitis & 711 & $123.3(4.3)$ & $\begin{array}{c}2.0 \\
(<0.001)\end{array}$ & - & \\
\hline & Stable periodontitis & 277 & $126.5(5.5)$ & $\begin{array}{c}5.2 \\
(<0.001)\end{array}$ & $\begin{array}{c}3.2 \\
(<0.001)\end{array}$ & - \\
\hline & Unstable periodontitis & 402 & $128.6(4.7)$ & $\begin{array}{c}7.3 \\
(<0.001)\end{array}$ & $\begin{array}{c}5.3 \\
(<0.001)\end{array}$ & $\begin{array}{c}2.1 \\
(<0.001)\end{array}$ \\
\hline \multirow[t]{4}{*}{ Unweighted } & Healthy & 840 & $123.0(4.0)$ & - & & \\
\hline & Gingivitis & 711 & $124.8(3.8)$ & $\begin{array}{c}1.8 \\
(<0.001)\end{array}$ & - & \\
\hline & Stable periodontitis & 277 & $130.9(4.6)$ & $\begin{array}{c}7.9 \\
(<0.001)\end{array}$ & $\begin{array}{c}6.1 \\
(<0.001)\end{array}$ & - \\
\hline & Unstable periodontitis & 402 & $132.4(4.0)$ & $\begin{array}{c}9.4 \\
(<0.001)\end{array}$ & $\begin{array}{c}7.6 \\
(<0.001)\end{array}$ & $\begin{array}{c}1.5 \\
(<0.001)\end{array}$ \\
\hline
\end{tabular}

Table footnote:

SBP: systolic blood pressure; SD: standard deviation 